\title{
Three-dimensional vision enhances task performance independently of the surgical method
}

\author{
O. J. Wagner • M. Hagen - A. Kurmann • \\ S. Horgan · D. Candinas $\cdot$ S. A. Vorburger
}

Received: 3 November 2011/Accepted: 2 April 2012/Published online: 12 May 2012

(C) Springer Science+Business Media, LLC 2012

\begin{abstract}
Background Within the next few years, the medical industry will launch increasingly affordable three-dimensional (3D) vision systems for the operating room (OR). This study aimed to evaluate the effect of two-dimensional (2D) and 3D visualization on surgical skills and task performance.

Methods In this study, 34 individuals with varying laparoscopic experience (18 inexperienced individuals) performed three tasks to test spatial relationships, grasping and positioning, dexterity, precision, and hand-eye and handhand coordination. Each task was performed in 3D using binocular vision for open performance, the Viking 3Di Vision System for laparoscopic performance, and the DaVinci robotic system. The same tasks were repeated in 2D using an eye patch for monocular vision, conventional laparoscopy, and the DaVinci robotic system.
\end{abstract}

O. J. Wagner · M. Hagen · A. Kurmann · D. Candinas ·

S. A. Vorburger

Department of Visceral and Transplantation Surgery, Inselspital,

University Hospital Bern and University of Bern, Bern,

Switzerland

e-mail: oliverwagner.md@gmail.com

O. J. Wagner · S. Horgan

Department of Surgery, Center for the Future of Surgery,

University of California, San Diego, CA, USA

M. Hagen

Division of Visceral Surgery, University Hospital Geneva,

Geneva, Switzerland

S. A. Vorburger $(\bowtie)$

Regional Hospital Emmental, Oberburgstrasse 54,

3400 Burgdorf, Switzerland

e-mail: stephan.vorburger@rs-e.ch
Results Loss of 3D vision significantly increased the perceived difficulty of a task and the time required to perform it, independently of the approach $(P<0.0001-$ 0.02). Simple tasks took $25 \%$ to $30 \%$ longer to complete and more complex tasks took $75 \%$ longer with $2 \mathrm{D}$ than with 3D vision. Only the difficult task was performed faster with the robot than with laparoscopy $(P=0.005)$. In every case, 3D robotic performance was superior to conventional laparoscopy (2D) $(P<0.001-0.015)$.

Conclusions The more complex the task, the more 3D vision accelerates task completion compared with $2 \mathrm{D}$ vision. The gain in task performance is independent of the surgical method.

Keywords 2D - 3D laparoscopy - 3D vision - Robotic surgery $\cdot$ Surgical skills $\cdot$ Task performance

In recent years, minimally invasive surgery has demonstrated benefits for easy to moderately complex surgical interventions compared with conventional open surgery [15]. These advantages and the continuous gain in experience have resulted in a willingness to perform more complex laparoscopic procedures $[1,6,7]$. Still, advanced laparoscopy is extremely challenging due to technical limitations [8], with the result that surgeons must undergo extensive training and experience flat learning curves to provide patients with safe, minimal access surgery. To allow for safe laparoscopy in more complex surgical areas and to ease potential novel endoscopic techniques (e.g., natural orifice transluminal endoscopic surgery [NOTES]), technical innovations in the field must keep up with surgeons' demands.

Besides general shortcomings, such as the fulcrum effect or decreased haptic feedback, and despite the technical 
limitations of instrument design, two-dimensional (2D) vision on a flat screen has been identified as a major disadvantage of laparoscopy compared with open surgery [911]. Major advances in the development of surgical video imaging has mainly concerned image quality, leading to bright, high-resolution images. However, improvement in depth perception by three-dimensional (3D) vision has been hampered by technical and financial limitations.

Experienced surgeons can compensate for the lack of the third dimension by using indirect clues such as the movement of the endoscope/motion parallax perspective, relative size, shading, texture gradient, familiar anatomy, and the size of anatomic structures [12,13]. With the advances in $3 \mathrm{D}$ technology evident in the increasingly popular $3 \mathrm{D}$ movies and surging 3D products in the home entertainment segment, the medical technology industry expects the emergence of affordable and high-quality 3D vision systems in the operating room on a large scale in the next 2-3 years (in analogy to high-definition [HD] products).

Several researchers have compared the role of 3D imaging with the traditional 2D mode during laparoscopy [14-29] and robotic surgery [30-38]. The advantages of 3D over $2 \mathrm{D}$ vision are consistent in robotic surgery, but the results for conventional laparoscopy differ greatly. Some studies have indicated equivalent task performance, whereas others have detected superior outcomes for some or all tasks performed using 3D vision. These differences appear to originate mainly from incoherent study designs and the use of inferior, earlier-generation 3D vision systems that provide video quality with low image resolution and only near real-time transmission. Thus, no definite conclusion can be drawn from the literature, especially for laparoscopy.

This study aimed to assess the overall role of 3D vision during surgical performance in open, laparoscopic, and robotic tasks. The use of an up-to-date 3D laparoscopic vision system allowed for a direct comparison with highresolution monitors.

\section{Materials and methods}

Participants and tasks

The difference between 3D and 2D vision was evaluated in 34 individuals with varying surgical experience. Only subjects with normal or corrected-to-normal vision were selected. The mean age of the 34 participants ( 20 men and 14 women) was 31.8 years (range, 23-47 years). The majority of the participants $(n=19)$ had fewer than 3 years of professional surgical experience, whereas 16 participants were already experienced laparoscopic surgeons. The inexperienced surgeons were either interns (last-year medical students) or first- and second- year surgical residents with an experience level of 20 or fewer basic minimally invasive procedures. The experienced surgeons were board-certified attending surgeons with a minimum of 100 minimally invasive surgical procedures.

Each individual performed three tasks (T1-T3) using open, laparoscopic, and robotic surgical techniques (Fig. 1). All the tasks were performed in 3D first, followed by the same tasks in $2 \mathrm{D}$ to exclude bias due to the learning effect. Each participant was instructed concerning the specific tasks to be performed. The participants were allowed to practice each task two times before registering the performance. All the participants performed the tasks in an identical sequence under identical conditions.

Three different skill pods ((The Chamberlain Group, Great Barrington, MA, USA) used for introducing practitioners to the skills required for minimally invasive surgery were used for the tasks (Fig. 1). Task 1 (T1) tested 3D imaging and spatial relationships. To test simple grasping and positioning maneuvers, we used the Sea Spike Pod containing soft cones of different sizes and shapes. Three small rubber rings were placed over one soft cone. The participants were required to grasp and distribute the rings one after another, placing them over three separate predetermined cones. Subsequently, the rings had to be transferred back to the initial cone.

Task 2 (T2) tested dexterity by suturing (without knottying) of a simulated gaping skin incision (Skin Suturing Pod). In this drill, three continuous stitches were placed in parallel.

Task 3 (T3) tested dexterity and precision using a suture with a curved needle (Vicryl 3-0; SH, Ethicon Inc., Cincinnati, OH, USA) that had to be passed from hand to hand through 10 small flexible eyelets. The eyelets were numbered and arranged in a S-curve and had to be passed in numeric order (S Hook Pod).

Each task required an appropriate amount of two-handed coordination and ambidexterity, which are considered essential for testing depth perception. Each task was evaluated by the time needed to complete the task. After the completion of each module (open, laparoscopic, robotic), the participants were asked to estimate the difficulty of the task on a visual analog scale (VAS) ranging from 1 (very easy) to 10 (extremely difficult to barely realizable).

\section{Imaging systems}

Each task was performed in both $3 \mathrm{D}$ and $2 \mathrm{D}$ vision (Fig. 1). Binocular vision was used to express true 3D vision for open performance, whereas monocular vision was achieved by covering one eye with an eye pad. Monocular vision should at least partly mimic a 2D 

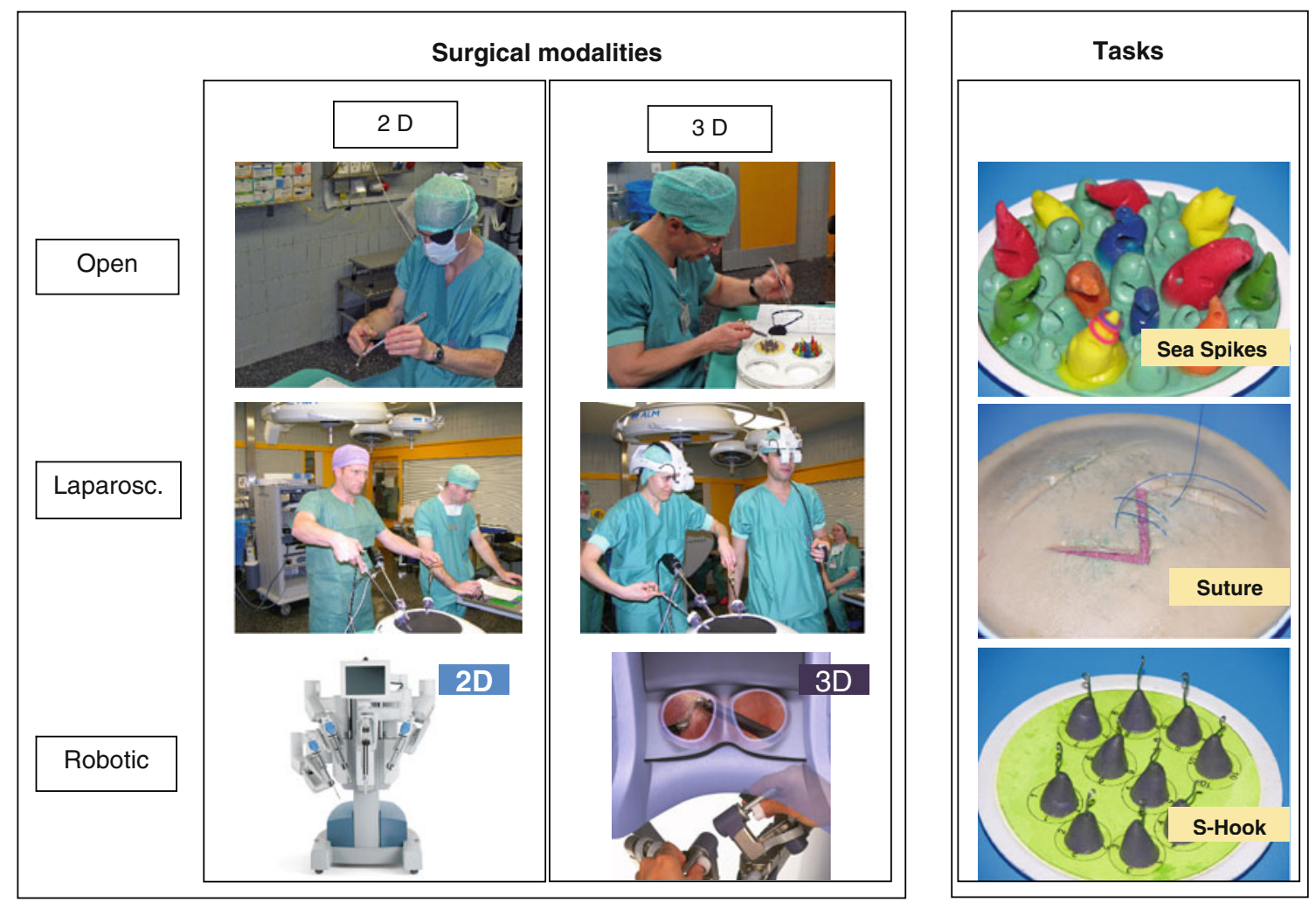

Fig. 1 Left The surgical modalities (open, laparoscopic, robotic) and the way that two-dimensional (2D) and 3D vision was implemented. The robotic DaVinci system allowed for direct switching between the 2D and 3D modes. Right The tasks to be performed are shown. Task 1: Sea Spike Pod for 3D imaging and spatial relationship testing. Task

environment by taking away binocular disparity, an important visual cue that estimates distance and provides information to the brain, in which depth perception is extracted from the two 2D retinal images.

The EndoSite 3Di Digital Vision System (Viking Systems, La Jolla, CA, USA) was used for 3D laparoscopic performance. This system couples a 3D view with a headmounted display, allowing spectral depth perception with the use of traditional laparoscopic instrumentation. The system includes a stereo digital scope (dual three-chip charge-coupled device [3CCD] optical channel) attached to a 3D data-processing unit, which conveys information to a head-mounted display. The head-mounted display consists of three liquid crystal displays (LCD) per eye (HD-SDI, 1080i monitor) attached to a headset, allowing for stereoscopic 3D vision.

The 2D laparoscopic system (Karl Storz, Tuttlingen, Germany) consisted of a standard laparoscopic video tower with a $10-\mathrm{mm}, 30^{\circ}$ scope and a 3CCD digital system attached to a 23-in. HD (1080i) flat screen video monitor. The optics were optimized in both systems before the performance of the task, and lighting was adjusted to similar levels for all systems. The laparoscope was adjusted and remained fixated during task performance. In all tasks,
2: Suture Pod for testing dexterity by suturing of a simulated gaping skin incision (without knot-tying). Task 3: S-Hook Pod for testing dexterity, precision, and manipulation with both hands (needle transfer)

the participants used the same laparoscopic instrumentation to complete the task.

The DaVinci S Surgical System (Intuitive Surgical Inc., Sunnyvale, CA, USA) was used for robotic surgical performance in the 3D or 2D mode. The InSite 3D endoscope (EndoSite 3Di Digital Vision System, Viking Sys-tems, La Jolla, CA, USA) provided two separate vision channels linked to two separate color monitors. The images were presented directly in the viewer on two continuous-tone cathode-ray tube monitors to produce a clear 3D image. The right and left eyes received separate images from each camera to a set focal point. Two 3CCD cameras with 800 lines of resolution were used. This vision system also incorporates the Intuitive Surgical image processing equipment, which is composed of high-performance video cameras and specialized edge enhancement and noise reduction equipment.

Statistical analysis

Student's $t$-test was used to test for differences between two groups, and one-way analysis of variance (ANOVA) was used to compare several groups. The Wilcoxon signedrank test was used for repeated measures. A $P$ value less 
than 0.05 was considered significant. Calculations were performed using NCSS 2001 (Number Cruncher Statistical Software, Kaysville UT, USA).

\section{Results}

Task performance in 2D was considered more difficult than in $3 \mathrm{D}$

The 34 participants were questioned about the subjective difficulty of the performed tasks. The tasks were rated immediately after completion of each task with each modality. The Sea Spike task was perceived as the easiest, whereas the S Hook task was rated as difficult (in 3D) to very difficult (in 2D). For all the tasks in all surgical modalities, the perceived difference in difficulty was significantly higher in 2D than in 3D (Table 1). The difference between 2D and 3D was independent of the laparoscopic experience. Generally, the open technique was considered the easiest way to complete a task. The laparoscopic modality was perceived as the most difficult way to solve a task, with robotic surgery having a slight advantage for experienced laparoscopic surgeons.

$3 \mathrm{D}$ vision allowed faster task performance than $2 \mathrm{D}$ vision

We used the task completion time for objective determination of task difficulty and individual participant performance. The open modality allowed for the fastest task completion, and 3D vision allowed for faster completion than $2 \mathrm{D}$ vision. The difference between $3 \mathrm{D}$ and $2 \mathrm{D}$ vision remained significant over all the tasks, independently of the surgical modality chosen $(\operatorname{Pmax}=0.02$, Wilcoxon signed rank test; Fig. 2).

All task performances started in $3 \mathrm{D}$ vision mode to eliminate improved performance due to the effect of training and learning. The time required to complete a task corresponded well with the perceived task difficulty.

The increased time to perform a task in 2D depended on task difficulty, not method

Two-dimensional vision reduced the speed at which tasks were completed by $19 \%$ to $88 \%$ (3D time was used as a reference, $100 \%$; Table 2). The performance time due to the loss of $3 \mathrm{D}$ vision increased $19 \%$ to $31 \%$ for the suture task, in which haptic feedback is a relevant factor (a stitch also can be performed blindfolded).

Time loss was more homogeneous than the other tasks, which depended predominantly on vision. The Sea Spike task, perceived as an easy task, took approximately onethird longer when vision was reduced to $2 \mathrm{D}$. The difference between $3 \mathrm{D}$ and $2 \mathrm{D}$ vision became more pronounced when the difficult S Hook Pod task was completed and led on the average to a $75 \%$ (range, 69-88 \%) time increase when $3 \mathrm{D}$ vision was lost. Interestingly, the additional time required to perform a task in $2 \mathrm{D}$ compared with $3 \mathrm{D}$ depended on the difficulty of the task itself and, to a much less extent, if at all, on the surgical method.

Table 1 Rating of task difficulty depending on experience and vision ${ }^{\mathrm{a}}$

\begin{tabular}{|c|c|c|c|c|c|c|}
\hline \multirow[t]{2}{*}{ Rating of tasks (VAS) } & \multicolumn{2}{|l|}{ Sea spikes } & \multicolumn{2}{|l|}{ Suture } & \multicolumn{2}{|l|}{ S Hooks } \\
\hline & $\begin{array}{l}\text { 2D } \\
\text { VAS }\end{array}$ & $3 \mathrm{D}$ & $\begin{array}{l}\text { 2D } \\
\text { VAS }\end{array}$ & $3 \mathrm{D}$ & $\begin{array}{l}\text { 2D } \\
\text { VAS }\end{array}$ & $3 \mathrm{D}$ \\
\hline \multicolumn{7}{|l|}{ Open } \\
\hline No exp & $2.8 \pm 1.2$ & $1.4 \pm 0.7$ & $3 \pm 1.0$ & $1.7 \pm 0.7$ & $4.1 \pm 1.5$ & $2.5 \pm 1.1$ \\
\hline Lap exp & $2.8 \pm 1.0$ & $1.3 \pm 0.7$ & $2.1 \pm 1.1$ & $1.1 \pm 0.3$ & $4.7 \pm 1.6$ & $2.6 \pm 1.3$ \\
\hline $2 \mathrm{D}$ vs $3 \mathrm{D}$ & $P<0.001$ & & $P<0.001$ & & $P<0.001$ & \\
\hline \multicolumn{7}{|l|}{ Laparoscopic } \\
\hline No exp & $5 \pm 1.4$ & $3.9 \pm 1.1$ & $6.6 \pm 1.4$ & $5.9 \pm 1.9$ & $8.5 \pm 1.3$ & $6.7 \pm 1.8$ \\
\hline Lap exp & $4.2 \pm 1.8$ & $2.9 \pm 1.2$ & $4.9 \pm 1.6$ & $3.7 \pm 1.2$ & $7.7 \pm 1.5$ & $5.7 \pm 1.6$ \\
\hline $2 \mathrm{D}$ vs $3 \mathrm{D}$ & $P<0.001$ & & $P=0.005$ & & $P<0.001$ & \\
\hline \multicolumn{7}{|l|}{ Robotic } \\
\hline No exp & $3 \pm 1.3$ & $2.28 \pm 1.4$ & $5.5 \pm 2.2$ & $4.7 \pm 2.3$ & $6.7 \pm 1.2$ & $5.2 \pm 1.7$ \\
\hline Lap exp & $3.9 \pm 1.8$ & $2.67 \pm 1.7$ & $4.6 \pm 1.7$ & $3.3 \pm 1.5$ & $6.5 \pm 1.3$ & $4.4 \pm 1.6$ \\
\hline $2 \mathrm{D}$ vs $3 \mathrm{D}$ & $P<0.002$ & & $P=0.005$ & & $P<0.001$ & \\
\hline
\end{tabular}

$2 D$ two-dimensional, $3 D$ three-dimensional, $V A S$ visual analog scale, No exp participant with no or minimal laparoscopic experience, Lap exp experienced laparoscopic surgeon

${ }^{a} P$ values are calculated for the difference in means between 2D and 3D ( $n=34 ; t$-test) 

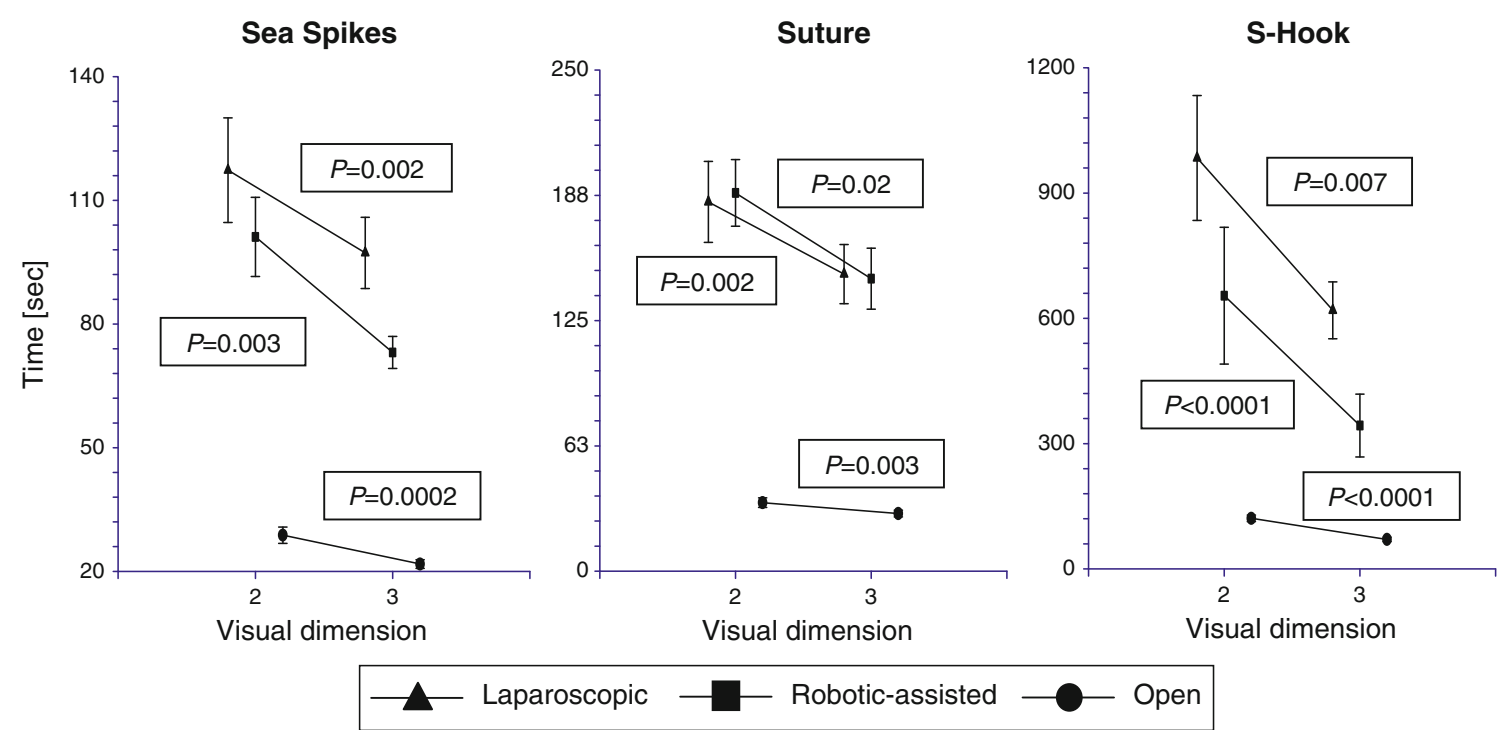

Fig. 2 Task completion is faster with three-dimensional (3D) vision than with $2 \mathrm{D}$ vision. The median time used to complete the three tasks for each of the surgical modalities is shown. The difference between the 2D and 3D times were calculated for each participant individually. Thus, the 2D and 3D medians are connected for better visualization of

Table 2 Difference in percentage of time required to complete a task in $2 \mathrm{D}$ compared with $3 \mathrm{D}$

\begin{tabular}{llll}
\hline Surgical modality & Sea spikes $(\%)$ & Suture $(\%)$ & S Hook pad (\%) \\
\hline Overall & 33 & 25 & 75 \\
Open & 32 & 19 & 69 \\
Laparoscopic & 30 & 28 & 71 \\
Robotic & 38 & 31 & 88
\end{tabular}

$2 D$ two-dimensional, $3 D$ three-dimensional

Robot-assisted task performance tends to be faster, independently of vision

For all tasks, the open surgical method remained significantly faster than the laparoscopic or robotic technique. The difference between laparoscopy and robotic surgery mainly consists of a reduced but preserved haptic feedback for laparoscopic instruments, with the DaVinci robot allowing for a greater range of motion.

We eliminated the influence of depth perception by comparing performance in $2 \mathrm{D}$ and $3 \mathrm{D}$. Only in the suture task, which requires haptic feedback to some extent, was laparoscopic performance comparable with the robotic performance (3D vision: mean laparoscopic time, $155.6 \mathrm{~s}$; $95 \%$ confidence interval $[\mathrm{CI}] 121.3-190 \mathrm{~s}$ vs mean robotic time, $142.3 \mathrm{~s} ; 95 \%$ CI, 107.5-177.1 s; $P>0.05$; Fig. 3). For tasks less influenced by haptic feedback, task completion in 3D was significantly faster with the robot than with laparoscopy (Sea Spike: laparoscopic mean time, the paired data. Error bars represent the standard deviation. All tasks were completed faster with the open modality. The $P$ values were calculated by the Wilcoxon signed-rank test for repeated measurements

$99.6 \mathrm{~s} ; 95 \% \mathrm{CI}, 78.1-121 \mathrm{~s}$ vs robotic mean time, $69.5 \mathrm{~s}$; $95 \%$ CI, 63.1-76 s; $P=0.004$; Fig. 3). For the more demanding S Hook task, the according means were $600 \mathrm{~s}$ (95\% CI, 429-770 s) for laparoscopic performance and $343 \mathrm{~s}$ (95\% CI, 186-500 s) for robotic performance ( $P=0.005$, Wilcoxon signed rank). Interestingly, the difference between the two methods was more pronounced in all tasks with 3D vision.

Robotic performance in 3D is superior to conventional laparoscopy in 2D

Subsequently, we addressed the difference in depth perception between conventional laparoscopy (typically 2D mode) and robotic surgery with the DaVinci system in 3D. All the tasks were performed significantly faster with the $3 \mathrm{D}$ robotic modality than with $2 \mathrm{D}$ laparoscopy. The mean time for the Sea Spike task was $121.8 \mathrm{~s}$ (95\% CI, 93.3-150.3 s) with laparoscopy and $70.8 \mathrm{~s}$ (95\% CI, 64.1-77.4 s) with robotic surgery $(P<0.0001)$. The mean times were $191.7 \mathrm{~s}$ (95\% CI, 149.1-234.3 s) versus 142.1 s (95 \% CI, 110.3-173.7 s); $P=0.015$, (Wilcoxon signed-rank test) for the suture task and $824.8 \mathrm{~s}(95 \% \mathrm{CI}$, 510.3-1139.4 s) versus $343.3 \mathrm{~s}(95 \% \mathrm{CI}, 186.5-500.1 \mathrm{~s}$ ); $P=0.0003$, (Wilcoxon signed-rank test) for the $\mathrm{S}$ Hook task. These findings put previous reports about the comparison of laparoscopic and robotic skills into perspective because the skills were compared under different visual conditions, namely, under $2 \mathrm{D}$ versus $3 \mathrm{D}$ vision. 


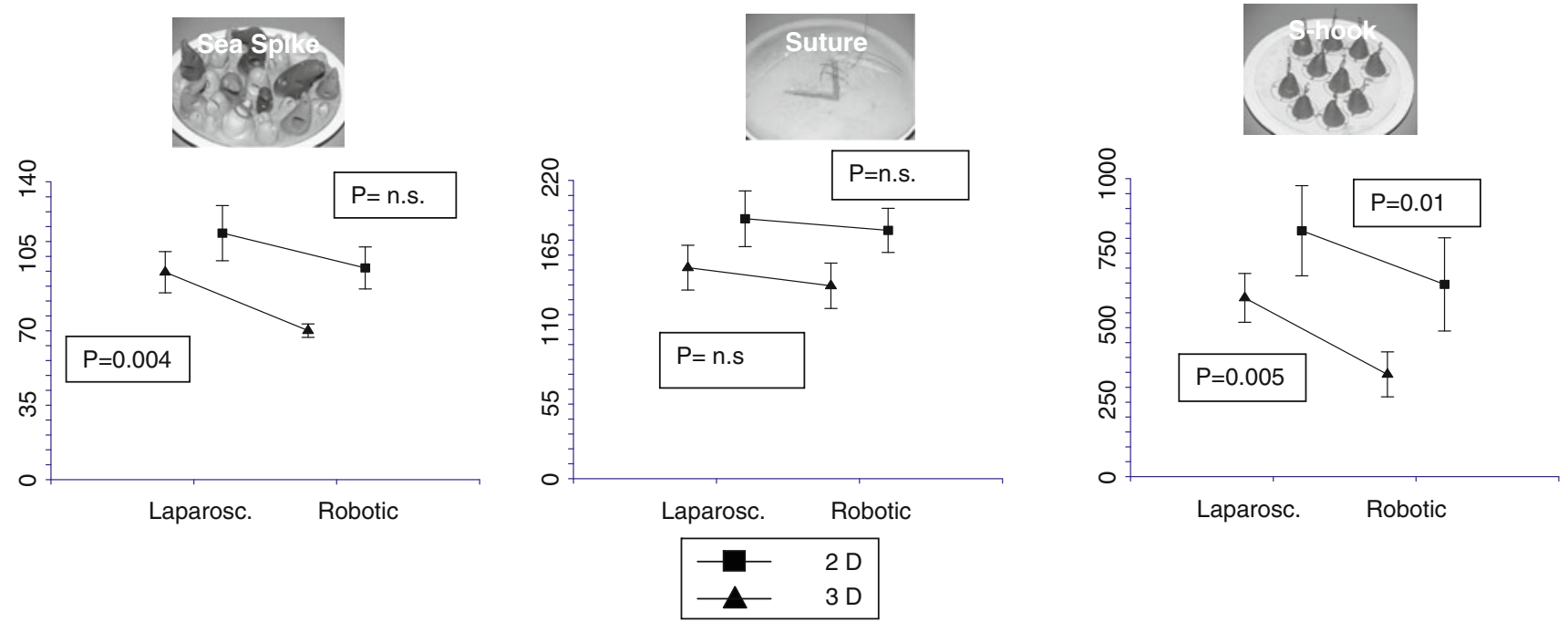

Fig. 3 A trend toward faster task completion with the robotic modality than with the laparoscopic modality was observed. The median times for task completion with the laparoscopic and robotassisted modalities are shown (both two-dimensional [2D] and 3D vision are shown). The medians are connected to emphasize the paired analysis of each participant's performance in the two

\section{Discussion}

The data presented show that task performance with $3 \mathrm{D}$ visualization is superior to that with $2 \mathrm{D}$ visualization independently of participants' laparoscopic experience, the difficulty of the task, or the surgical modality.

Although 3D visualization is intuitively considered an important and contributing factor for improved performance during minimally invasive surgery, publications comparing $2 \mathrm{D}$ and $3 \mathrm{D}$ vision in the last two decades have reported contradictory results [14-24, 33, 39, 40].

When laparoscopic skills are assessed, two major requirements seem to be paramount: ability to translate information received from a $2 \mathrm{D}$ image to the $3 \mathrm{D}$ visceral organ situs (visiospatial translation and perception) and psychomotor hand-eye coordination [41]. To reach an expert level, the acquisition of both skills demands sustained and deliberate practice over years. Thus, learning curves for complex and advanced conventional laparoscopy are flat and slower compared with those for open surgery, requiring extensive training and experience [42].

Our findings corroborate previous studies describing the advantage of using the 3D mode over the 2D mode during robotic surgery and the superiority of advanced 3D optical systems over conventional 2D laparoscopy. Conflicting findings in several other studies are most likely the result of using less efficient 3D vision systems. The technical quality of $3 \mathrm{D}$ vision systems appears to have a drastic effect on overall task performance. It is not surprising that a significant difference between $2 \mathrm{D}$ and $3 \mathrm{D}$ vision was modalities. Error bars represent the standard deviation. For the suture task, haptic feedback was considered helpful for completion of the task. The difficult S Hook task took considerably more time with laparoscopic surgery than with robot-assisted surgery $(P$ values were calculated using the Wilcoxon signed-rank test)

observed in our studies because we used modern highdefinition 3D systems that had already been tested in clinical practice. Not only was task completion time accelerated, but the tasks were perceived as significantly easier with $3 \mathrm{D}$ vision than with $2 \mathrm{D}$ vision.

Another interesting aspect of this study was the assessment of robot-assisted performance versus laparoscopy independently of the difference in visual dimensions. Conventional laparoscopy relies on 2D vision, whereas robotic surgery, using the only commercially available Food and Drug Administration (FDA)-approved robotic system, is performed in $3 \mathrm{D}$ vision.

A direct comparison of laparoscopy with robotic surgery found that the robotic surgical system allows steepening of the learning curve for almost any laparoscopic procedure [34, 37]. This improvement was attributed to superior ergonomics and enhanced dexterity, precision, and control, as well as to improved 3D visualization. However, we did not investigate the extent to which the additional depth perception accounted for the better performance.

Our results showed little to no difference between robotassisted and laparoscopic performance for easy tasks, especially if a certain degree of haptic feedback is helpful for task completion. For difficult tasks, 3D vision and robotic assistance resulted in a faster performance compared with laparoscopy. Given that $3 \mathrm{D}$ robotic performance was significantly superior to 2D laparoscopic performance, the most important difference in task performance between conventional laparoscopy and robot-assisted surgery is vision. This is especially true when spatial limitations are 
absent. This importance for 3D vision has been suggested repeatedly by other researchers [12, 32, 35, 37].

Arguably, the most interesting finding of this study was that $3 \mathrm{D}$ vision improved the task completion speed according to the difficulty of the task. The modality in which the task was performed played no major role. In other words, regardless of the surgical approach chosen, the loss of $3 \mathrm{D}$ vision delayed the completion of a task proportionally to the difficulty of the task. This finding should translate directly into clinical practice. For more demanding procedures, the gain in operating time would favor the use of a vision system with depth perception independently of the surgeon's experience. An efficient 3D optical system would facilitate advanced laparoscopic surgery and increase performance speed by $60-70 \%$.

In addition to the reduction in surgical stress due to a reduction in the perceived difficulty of the intervention, it is likely that improved task performance during laparoscopy also would lower complication rates and the necessity for conversion to open surgery. Image quality has gained a major focus in the current laparoscopic field, but the role of depth perception with 3D vision still is underestimated in everyday surgery. The future integration of 3D systems will facilitate the expansion of laparoscopic surgery to more complex interventions and help to advance the field of endoscopically assisted surgery, notably NOTES procedures.

Disclosures Monika E. Hagen receives a salary from Intuitive Surgical Inc. However, the financial relation started after the current study was completed. O. J. Wagner, Anita Kurmann, S. Horgan, Daniel Candinas, and Stephan A. Vorburger have no conflicts of interest or financial ties to disclose.

\section{References}

1. Dagher I, Di Giuro G, Dubrez J, Lainas P, Smadja C, Franco D (2009) Laparoscopic versus open right hepatectomy: a comparative study. Am J Surg 198:173-177

2. Keus F, de Jong JA, Gooszen HG, van Laarhoven CJ (2006) Laparoscopic versus open cholecystectomy for patients with symptomatic cholecystolithiasis. Cochrane Database Syst Rev (4):CD006231

3. Laine S, Rantala A, Gullichsen R, Ovaska J (1997) Laparoscopic vs conventional Nissen fundoplication: a prospective randomized study. Surg Endosc 11:441-444

4. Medeiros LR, Stein AT, Fachel J, Garry R, Furness S (2008) Laparoscopy versus laparotomy for benign ovarian tumor: a systematic review and meta-analysis. Int J Gynecol Cancer 18: 387-399

5. Sauerland S, Lefering R, Neugebauer EA (2004) Laparoscopic versus open surgery for suspected appendicitis. Cochrane Database Syst Rev (4):CD001546

6. Avital S, Zundel N, Szomstein S, Rosenthal R (2005) Laparoscopic transhiatal esophagectomy for esophageal cancer. Am J Surg 190(1):69-74
7. Sinha CK, Paramalingam S, Patel S, Davenport M, Ade-Ajayi N (2009) Feasibility of complex minimally invasive surgery in neonates. Pediatr Surg Int 25:217-221

8. Heemskerk J, Zandbergen R, Maessen JG, Greve JW, Bouvy ND (2006) Advantages of advanced laparoscopic systems. Surg Endosc 20:730-733

9. Gallagher AG, Ritter EM, Lederman AB, McClusky DA III, Smith CD (2005) Video-assisted surgery represents more than a loss of three-dimensional vision. Am J Surg 189:76-80

10. Hubber JW, Taffinder N, Russell RC, Darzi A (2003) The effects of different viewing conditions on performance in simulated minimal access surgery. Ergonomics 46:999-1016

11. Risucci D, Geiss A, Gellman L, Pinard B, Rosser J (2001) Surgeon-specific factors in the acquisition of laparoscopic surgical skills. Am J Surg 181:289-293

12. Byrn JC, Schluender S, Divino CM, Conrad J, Gurland B, Shlasko E, Szold A (2007) Three-dimensional imaging improves surgical performance for both novice and experienced operators using the da Vinci Robot System. Am J Surg 193:519-522

13. Goldstein E (2003) Blackwell handbook of sensation and perception, 6th edn. Wiley, Engelska

14. Bhayani SB, Andriole GL (2005) Three-dimensional (3D) vision: does it improve laparoscopic skills? An assessment of a 3D headmounted visualization system. Rev Urol 7:211-214

15. Buess GF, van Bergen P, Kunert W, Schurr MO (1996) Comparative study of various $2-\mathrm{D}$ and 3 -D vision systems in minimally invasive surgery. Chirurg 67:1041-1046

16. Chan AC, Chung SC, Yim AP, Lau JY, Ng EK, Li AK (1997) Comparison of two-dimensional vs three-dimensional camera systems in laparoscopic surgery. Surg Endosc 11:438-440

17. Dion YM, Gaillard F (1997) Visual integration of data and basic motor skills under laparoscopy: influence of 2-D and 3-D videocamera systems. Surg Endosc 11:995-1000

18. Hanna GB, Cuschieri A (2000) Influence of two-dimensional and three-dimensional imaging on endoscopic bowel suturing. World J Surg 24:444-448 discussion 448-449

19. Hanna GB, Shimi SM, Cuschieri A (1998) Randomised study of influence of two-dimensional versus three-dimensional imaging on performance of laparoscopic cholecystectomy. Lancet 351: 248-251

20. Herron DM, Lantis JC II, Maykel J, Basu C, Schwaitzberg SD (1999) The 3-D monitor and head-mounted display: a quantitative evaluation of advanced laparoscopic viewing technologies. Surg Endosc 13:751-755

21. Jones DB, Brewer JD, Soper NJ (1996) The influence of threedimensional video systems on laparoscopic task performance. Surg Laparosc Endosc 6:191-197

22. McDougall EM, Soble JJ, Wolf JS Jr, Nakada SY, Elashry OM, Clayman RV (1996) Comparison of three-dimensional and twodimensional laparoscopic video systems. J Endourol 10:371-374

23. Peitgen K, Walz MV, Walz MV, Holtmann G, Eigler FW (1996) A prospective randomized experimental evaluation of three-dimensional imaging in laparoscopy. Gastrointest Endosc 44:262-267

24. Pietrabissa A, Scarcello E, Carobbi A, Mosca F (1994) Threedimensional versus two-dimensional video system for the trained endoscopic surgeon and the beginner. Endosc Surg Allied Technol 2: $315-317$

25. Taffinder N, Smith SG, Huber J, Russell RC, Darzi A (1999) The effect of a second-generation 3D endoscope on the laparoscopic precision of novices and experienced surgeons. Surg Endosc 13: 1087-1092

26. Tevaearai HT, Mueller XM, von Segesser LK (2000) 3-D vision improves performance in a pelvic trainer. Endoscopy 32:464-468

27. Thomsen MN, Lang RD (2004) An experimental comparison of 3-dimensional and 2-dimensional endoscopic systems in a model. Arthroscopy 20:419-423 
28. van Bergen P, Kunert W, Bessell J, Buess GF (1998) Comparative study of two-dimensional and three-dimensional vision systems for minimally invasive surgery. Surg Endosc 12:948-954

29. Votanopoulos K, Brunicardi FC, Thornby J, Bellows CF (2008) Impact of three-dimensional vision in laparoscopic training. World J Surg 32:110-118

30. Badani KK, Bhandari A, Tewari A, Menon M (2005) Comparison of two-dimensional and three-dimensional suturing: Is there a difference in a robotic surgery setting? J Endourol 19:1212-1215

31. Blavier A, Gaudissart Q, Cadiere GB, Nyssen AS (2006) Impact of $2 \mathrm{D}$ and $3 \mathrm{D}$ vision on performance of novice subjects using da Vinci robotic system. Acta Chir Belg 106:662-664

32. Blavier A, Gaudissart Q, Cadiere GB, Nyssen AS (2007) Comparison of learning curves and skill transfer between classical and robotic laparoscopy according to the viewing conditions: implications for training. Am J Surg 194:115-121

33. Falk V, Mintz D, Grunenfelder J, Fann JI, Burdon TA (2001) Influence of three-dimensional vision on surgical telemanipulator performance. Surg Endosc 15:1282-1288

34. Hubens G, Coveliers H, Balliu L, Ruppert M, Vaneerdeweg W (2003) A performance study comparing manual and robotically assisted laparoscopic surgery using the da Vinci system. Surg Endosc 17:1595-1599

35. Jourdan IC, Dutson E, Garcia A, Vleugels T, Leroy J, Mutter D, Marescaux J (2004) Stereoscopic vision provides a significant advantage for precision robotic laparoscopy. Br J Surg 91: 879-885
36. LaGrange CA, Clark CJ, Gerber EW, Strup SE (2008) Evaluation of three laparoscopic modalities: robotics versus three-dimensional vision laparoscopy versus standard laparoscopy. J Endourol 22:511-516

37. Moorthy K, Munz Y, Dosis A, Hernandez J, Martin S, Bello F, Rockall T, Darzi A (2004) Dexterity enhancement with robotic surgery. Surg Endosc 18:790-795

38. Munz Y, Moorthy K, Dosis A, Hernandez JD, Bann S, Bello F, Martin S, Darzi A, Rockall T (2004) The benefits of stereoscopic vision in robotic-assisted performance on bench models. Surg Endosc 18:611-616

39. Mueller MD, Camartin C, Dreher E, Hanggi W (1999) Threedimensional laparoscopy: gadget or progress? A randomized trial on the efficacy of three-dimensional laparoscopy. Surg Endosc 13:469-472

40. Patel HR, Ribal MJ, Arya M, Nauth-Misir R, Joseph JV (2007) Is it worth revisiting laparoscopic three-dimensional visualization? A validated assessment. Urology 70:47-49

41. Hasson HM, Jason H (2007) Information retention and skill acquisition after CME meetings. Laparosc Today 6:4-6

42. Vickers AJ, Savage CJ, Hruza M, Tuerk I, Koenig P, MartinezPineiro L, Janetschek G, Guillonneau B (2009) The surgical learning curve for laparoscopic radical prostatectomy: a retrospective cohort study. Lancet Oncol 10:475-480 